\title{
Allergic Rhinitis in Preschool Children and the Clinical Utility of FeNO
}

\author{
Jisun Yoon, ${ }_{1}$ Yean Jung Choi, ${ }^{1}$ Eun Lee, ${ }_{1}^{2}$ Hyun-Ju Cho, ${ }^{1}$ Song-I Yang, ${ }_{1}$ Young-Ho Kim, ${ }_{1}^{4}$ Young-Ho Jung, ${ }_{1}^{5}$ Ju-Hee Seo, ${ }^{6}$ \\ Ji-won Kwon, ${ }_{1}^{7}$ Hyo-Bin Kim, ${ }_{1}^{8}$ So-Yeon Lee, ${ }^{3}$ Bong-Seong Kim, ${ }_{1}^{9}$ Jung Yeon Shim, ${ }_{1}^{10}$ Eun-Jin Kim, ${ }_{1}^{11}$ Joo-Shil Lee, ${ }^{11}$ \\ Soo-Jong Hong ${ }^{1 *}$
}

\author{
'Department of Pediatrics, Childhood Asthma Atopy Center, Research Center for the Standardization of Allergic Diseases, University of Ulsan College of \\ Medicine, Asan Medical Center, Seoul, Korea \\ ${ }^{2}$ Department of Pediatrics, Chonnam National University Hospital, Gwangju, Korea \\ ${ }^{3}$ Department of Pediatrics, Hallym University Sacred Heart Hospital, Hallym University College of Medicine, Anyang, Korea \\ ${ }^{4}$ Department of Pediatrics, Gyeongsang National University Changwon Hospital, Gyeongsang, Korea \\ ${ }^{5}$ Department of Pediatrics, CHA Bundang Medical Center, College of Medicine, CHA University, Seongnam, Korea \\ ${ }^{6}$ Department of Pediatrics, Dankook University Hospital, Cheonan, Korea \\ 'Department of Pediatrics, Seoul National University Bundang Hospital, Seongnam, Korea \\ ${ }^{8}$ Department of Pediatrics, Inje University Sanggye Paik Hospital, Inje University College of Medicine, Seoul, Korea \\ ${ }^{9}$ Department of Pediatrics, Gangneung Asan Hospital, Gangneung, Korea \\ ${ }^{10}$ Department of Pediatrics, Kangbuk Samsung Hospital, Sungkyunkwan University School of Medicine, Seoul, Korea \\ ${ }^{11}$ Division of Allergy and Chronic Respiratory Diseases, Center for Biomedical Sciences, Korea National Institute of Health, Korea Center for Disease Control and \\ Prevention, Cheongju, Korea
}

\footnotetext{
This is an Open Access article distributed under the terms of the Creative Commons Attribution Non-Commercial License (http://creativecommons.org/licenses/by-nc/4.0/) which permits unrestricted non-commercial use, distribution, and reproduction in any medium, provided the original work is properly cited.
}

Purpose: The nature of allergic rhinitis (AR) in preschool aged children remains incompletely characterized. This study aimed to investigate the prevalence of AR and its associated risk factors in preschool-aged children and to assess the clinical utility of fractional exhaled nitric oxide (FeNO). Methods: This general population-based, cross-sectional survey included 933 preschool-aged (3- to 7-year-old) children from Korea. Current AR was defined as having nasal symptoms within the last 12 months and physician-diagnosed AR. Results: The prevalence of current AR in preschool children was 17.0\% (156/919). Mold exposure (adjusted odds ratio [aOR], 1.67; 95\% confidence interval [Cl], 1.15-2.43) and the use of antibiotics (aOR, 1.97; 95\% Cl, 1.33-2.90) during infancy were associated with an increased risk of current $\mathrm{AR}$, whereas having an older sibling (a0R, 0.52; 95\% $\mathrm{Cl}, 0.35-0.75)$ reduced the risk. Children with current atopic AR had significantly higher geometric mean levels of FeNO compared to those with nonatopic rhinitis (12.43; range of 1standard deviation [SD], 7.31-21.14 vs 8.25; range of 1SD, 5.62-12.10, $P=0.001$ ) or non-atopic healthy children (8.58; range of $1 S D, 5.51-13.38, P<0.001)$. The FeNO levels were higher in children with current atopic AR compared with atopic healthy children (9.78; range of 1SD, 5.97-16.02, $P=0.083$ ). Conclusions: Mold exposure and use of antibiotics during infancy increases the risk of current $A R$, whereas having an older sibling reduces it. Children with current atopic AR exhibit higher levels of FeNO compared with non-atopic rhinitis cases, suggesting that FeNO levels may be a useful discriminatory marker for subtypes of AR in preschool children.

Key Words: Rhinitis; allergic; children; preschool; prevalence; risk factor; nitric oxide

\section{INTRODUCTION}

Allergic rhinitis (AR) is a common chronic disease in childhood and adolescence. ${ }^{1,2}$ AR negatively impacts physical, social, and psychological well-being, and these effects can also extend to other family members. ${ }^{1,3}$ Children with AR complain of disruptive sneezing, itching, watery rhinorrhea, and nasal blockage; these symptoms can indirectly cause sleep distur-
Correspondence to: Soo-Jong Hong, MD, PhD, Department of Pediatrics, Childhood Asthma Atopy Center, Research Center for the Standardization of Allergic Diseases, Ulsan University College of Medicine, Asan Medical Center, 88 Olympic-ro 43-gil, Songpa-gu, Seoul 05505, Korea.

Tel: +82-2-3010-3379; Fax: +82-2-473-3725; E-mail: sjhong@amc.seoul.kr Received: April 2, 2016; Revised: October 31, 2016; Accepted: December 19, 2016

- There are no financial or other issues that might lead to conflict of interest. 
bances and daily fatigue, thereby resulting in impaired school performance. ${ }^{4}$

The prevalence of AR is increasing worldwide. ${ }^{5}$ The International Study of Asthma and Allergies in Childhood (ISAAC) I studies revealed that the median average prevalence of rhinoconjunctivitis in the past year for 6- to 7-year-old children was $6.9 \%$ (range $0.8 \%-14.9 \%$ ) in $1997 .{ }^{6}$ ISSAC III studies (1999-2004) reported an average prevalence of current rhinoconjunctivitis symptoms of $8.5 \%$ (range $2.8 \%-21.8 \%$ ) in 6- to 7-year-old children and $14.6 \%(1.0 \%-45.0 \%)$ in 13 - to 14 -year-old children. ${ }^{7}$ The prevalence of AR in early childhood has continually increased from 1995 to 2008 in Seoul, Korea. Indeed, the prevalence of current AR was $11.9 \%$ in $1995,14.2 \%$ in $2000,19.4 \%$ in 2005 , and $22.2 \%$ in $2008 .^{8}$

By contrast, limited data on the prevalence of AR are available because of difficulties in the diagnosis or definition of AR, especially in preschool-aged children. In a study from central China, the prevalence of AR in 3- to 6-year-old children was reported to be $10.8 \%$ based on the diagnostic criteria of nasal symptoms and positive skin prick test (SPT) results. ${ }^{9}$ The prevalence of rhinitis symptoms ever in life time was reported in another study to be $43.4 \%$, whereas the prevalence of physician-diagnosed AR was $26.4 \%$ and $26.1 \%$ of 3 - to 5 -year old children were treated because of AR. ${ }^{5}$ Most studies of the prevalence of AR in preschool children have been performed using a questionnaire; therefore, the wide range of reports of the prevalence of AR may be partially attributed to an overestimation of AR. Furthermore, the prevalence of AR can be varied and depend upon the definition of AR.

Rhinitis is defined as inflammation of the nasal epithelium and characterized by at least 2 of the following nasal symptoms: rhinorrhea, blockage, sneezing, or itching. AR is caused by exposure to an allergen to which an individual is sensitized..$^{10}$ In preschool-aged children, it is challenging to distinguish AR from infectious rhinitis and to define AR because of low rates of allergen sensitization. ${ }^{3,11}$

The fractional concentration of exhaled nitric oxide (FeNO) is a noninvasive parameter used to measure airway inflammation by analyzing gas collected from a single breath. ${ }^{12} \mathrm{Up}$-regulation of inducible nitric oxide synthase during intranasal and airway inflammation can increase FeNO levels. ${ }^{13}$ FeNO is correlated with other markers of inflammation, such as total serum immunoglobulin E (IgE) and eosinophil counts in the airway mucosa, and its levels are elevated in asthmatic patients. ${ }^{12}$ Because allergic diseases, such as AR and atopic dermatitis (AD), can confound the relationship between FeNO and asthma, AR should be considered in the assessments of FeNO levels in patients with asthma. ${ }^{14}$ Additionally, subjects with AR who do not exhibit asthmatic symptoms can have significantly higher levels of FeNO than those in healthy school-aged children (15.3 vs 5.9 ppb). ${ }^{15}$ However, the levels of FeNO in preschool children with AR have not yet been reported and little is known about the ep- idemiology of AR in preschool-aged children.

This study aimed to investigate the prevalence and associated risk factors of AR in preschool-aged children (3- to 7-years-old). Additionally, we compared FeNO levels in children with AR according to the presence or absence of potential confounding factors, such as comorbidities related to asthma.

\section{MATERIALS AND METHODS}

\section{Study participants}

The present study was designed as a cross-sectional and community-based study as a part of a standardization study for the diagnosis and treatment of pediatric allergic diseases. It included 16 randomly selected childcare centers from Seoul and Gyeonggi province in Korea, between July and August of 2010. A modified ISAAC questionnaire ${ }^{2}$ was used to evaluate the prevalence of allergic diseases.

The study population included 480 boys and 453 girls (mean age, $4.87 \pm 1.05$ years). Patient demographic profiles and general information are presented in Table 1. A parental history of

Table 1. Subject characteristics

\begin{tabular}{lc}
\hline Characteristics & No./total No. (\%)* \\
\hline No. of subject & 933 \\
Responded to questionnaire & $919 / 933(98.5)$ \\
Age, mean \pm SD (year) & $4.87 \pm 1.05$ \\
Sex (M/F) & $480 / 453$ \\
BMI, mean \pm SD (kg/m²) & $15.9 \pm 1.65$ \\
Parental history of allergic disease & $449 / 916(49.0)$ \\
Parental history of asthma & $46 / 914(5.0)$ \\
Parental history of AR & $403 / 915(44.0)$ \\
Parental history of AD & $105 / 915(11.5)$ \\
Environmental tobacco smoking & $412 / 911(45.2)$ \\
Maternal education level & \\
$<$ College degree & $240 / 905(26.5)$ \\
$\geq$ College degree & $665 / 905(73.5)$ \\
Economic status (monthly income, USD) & \\
Low (<3,000) & $328 / 890(36.9)$ \\
Middle (3,000-5,000) & $341 / 890(38.3)$ \\
High ( $\geq 5,000)$ & $221 / 890(24.8)$ \\
Biomarker & \\
Eosinophil, mean $\pm S D(\%)$ & $4.17 \pm 3.20$ \\
Total lgE, mean $\pm S D(I U / m L)$ & $194.35 \pm 376.47$ \\
Atopy & $147 / 659(22.3)$ \\
FeNO, mean $\pm S D(p p b)$ & $10.31 \pm 6.51$ \\
\hline
\end{tabular}

SD, standard deviation; $M$, male; $F$, female; $B M I$, body mass index; $A R$, allergic rhinitis; AD, atopic dermatitis; USD, US dollar; IgE, immunoglobulin E; FeNO, fractional exhaled nitric oxide; SPT, skin prick test.

${ }^{*}$ Data are presented as number/total number (percentage) of patients, unless otherwise indicated; 'Defined as at least 1 positive SPT result (an allergen wheal diameter greater than a histamine wheal diameter and a histamine wheal diameter $\geq 3 \mathrm{~mm}$ ). 
any allergic disease, including asthma, $\mathrm{AR}$, or $\mathrm{AD}$, was reported for $49.0 \%$ of individuals, while a parental history of AR was noted for $44.0 \%$. Exposure to environmental tobacco smoke was reported in $45.2 \%$ of the study population.

The Institutional Review Board (2010-02 CON-14-P) of Asan Medical Center approved the study protocol. Written consent was obtained from all parents and guardians following a detailed explanation of the study.

\section{ISAAC questionnaire}

A modified Korean version of the ISAAC was previously validated as a tool for the assessment and diagnosis of allergic symptoms in Korean children. ${ }^{16}$ The questionnaire includes 3 main sections: (1) general patient characteristics, including name, sex, date of birth, height, and weight; (2) a history of symptoms related to asthma, $\mathrm{AR}, \mathrm{AD}$, allergic conjunctivitis, and food allergy; and (3) exposure to environmental factors associated with allergic disease. The Korean version of the ISAAC questionnaire was completed by the parents or guardians of the preschool-aged children.

\section{Prevalence of AR}

We determined the prevalence of the following factors: (1) sneezing or a runny/blocked nose at any point in life (i.e., AR symptoms ever); (2) sneezing or runny/blocked nose and AR symptoms within the 12 months prior to survey completion (i.e., AR symptoms in the last 12 months; (3) a diagnosis of AR at any point in a lifetime (i.e., AR diagnosis ever); (4) treatment for AR within 12 months prior to survey completion (i.e., AR treatment in the last 12 months); (5) exhibiting AR symptoms within the last 12 months and a diagnosis of AR by clinicians at any point in life (i.e., current AR); (6) exhibiting AR symptoms within the last 12 months and a diagnosis of AR by clinicians at any point in life along with atopy (i.e., current atopic AR); and (7) AR symptoms in the last 12 months who had 1 or more positive reactions on SPTs (i.e., atopic AR).

White blood cells (WBCs), eosinophils, total $\lg \mathrm{E}$, and specific $\lg \mathrm{E}$ WBCs and the percentage of blood eosinophils were measured. The levels of total serum IgE and specific IgE were measured by fluorescent enzyme immunoassay using the ImmunoCAP system (Phadia AB, Uppsala, Sweden). Titers of specific IgE were measured for 7 major inhaled allergens: Dermatophagoides farina (Der f), cat, dog, cockroach, Alternaria alternate, mugwort, and alder. The titers of specific IgE were considered to be positive if they were greater than $0.35 \mathrm{kUA} / \mathrm{L}$.

\section{SPTs}

SPTs were performed against the following 18 common aeroallergens and food allergens (Allergopharma, Reinbek, Germany): Dermatophagoides pteronyssinus (Der $p$ ), Der $f$, cat, dog, mugwort, grass, birch, ragweed, alder, oak, Japanese hop, As- pergillus fumigatus, Alternaria alternate, cockroach, milk, soybean, egg white, and peanut. As positive and negative controls, histamine, and isotonic saline, respectively, were used. Maximum wheal diameters that were greater than both of those caused by histamine and $3 \mathrm{~mm}$ in the absence of any reaction to saline were considered positive responses on SPTs after 15 minutes. Atopy was defined as exhibiting a positive response against at least 1 allergen on SPTs.

\section{FeNO}

The Levels of FeNO were measured using a Niox Mino analyzer (Aerocrine, Solna, Sweden) according to American Thoracic Society/European Respiratory Society (ATS/ERS) recommendations by a single experienced operator. ${ }^{17}$ Measurements were made at an expiratory flow rate of $50 \mathrm{~mL} / \mathrm{sec}$ and the duration of exhalation was at least 6 seconds to ensure that NO levels were stable. A total of 3 recorded FeNO measurements with repeated measurements within $10 \%$ of the mean were obtained for each subject. The mean value of the 3 measurements was recorded as the final FeNO. ${ }^{17}$

\section{Statistical analysis}

Statistical analyses were performed using SAS version 9.0 (SAS Institute, Cary, NC, USA). The prevalence of allergic diseases is presented as mean values with the $95 \%$ confidence interval (CI). To identify potential risk factors for current $\mathrm{AR}, \chi^{2}$ tests and logistic regression analyses were conducted. For multivariate analysis, age, sex, and body mass index (BMI) were adjusted as personal factors, a parental history of $\mathrm{AD}$ was adjusted as a genetic factor, and the level of maternal education was considered as a socioeconomic factor. Sensitization to Der $p$ or $\operatorname{Der} f$ indicated a positive response on SPTs or ImmunoCAP for house dust mite antigen. A $P$ value of $<0.05$ was used to define statistically significant differences.

\section{RESULTS}

\section{Prevalence of AR}

The prevalence of allergic diseases, including current AR, is shown in Fig. 1. Generally, an increasing trend was observed for the prevalence of AR with age in preschool-aged children. The rates of prevalence of AR symptoms ever in life, AR symptoms in the last 12 months, AR diagnosis in the life time, and AR treatment in the life time in 3- to 7-year-old children were $43.8 \%, 38.5 \%, 23.8 \%$, and $18.6 \%$, respectively. The prevalence of current AR, current atopic AR, and atopic AR symptoms within 12 months in 3- to 7-year-old children was $17.0 \%, 4.7 \%$, and $7.5 \%$, respectively (Fig. 1).

\section{Risk factors for current $A R$}

The independent risk factors for current AR included a history of asthma diagnosis (adjusted odds ratio [aOR], 2.96; 95\% CI, 


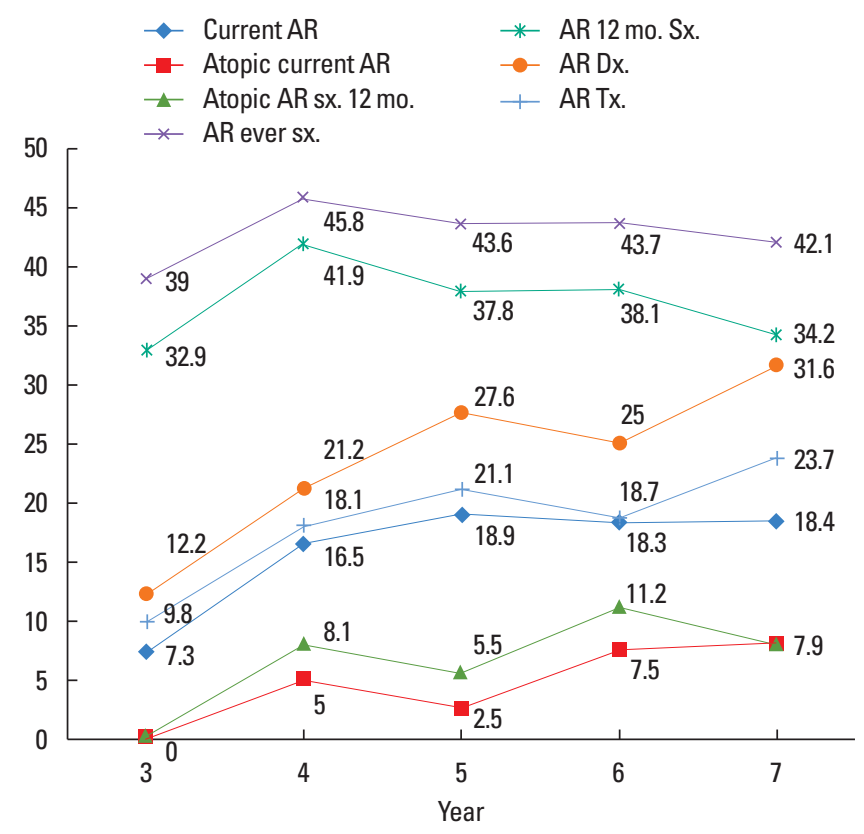

Fig. 1. The prevalence of all related $A R$ questionnaires in 3- to 7-year-old children. AR, allergic rhinitis; sx., symptom; 12 mo., within 12 months; Dx., diagnosis; Tx., treatment.
1.73-5.08), a history of wheezing in the last 12 months (aOR, 2.09; 95\% CI, 1.21-3.59), a history of $\mathrm{AD}$ diagnosis (aOR, 1.61; $95 \% \mathrm{CI}, 1.11-2.34$ ), and a parental history of asthma (aOR, 2.10; 95\% CI, 1.08-4.08). We also identified independent environmental risk factors for AR, which included the use of antibiotics in infancy for more than 3 days (aOR, 1.97; 95\% CI, 1.33-2.90) and exposure to indoor mold during infancy (aOR, 1.67; 95\% CI, 1.15-2.43). Additionally, having an older sibling reduced the risk of current AR (aOR, 0.52; 95\% CI, 0.35-0.75) (Table 2).

\section{Sensitization for current AR}

Table 3 shows analyses of associations between positive sensitization on SPTs and current AR. The independent risk factors for current AR included any any sensitization on SPTs (aOR, 1.99; 95\% CI, 1.26-3.14), $\operatorname{Der} p$ (aOR, 2.23; 95\% CI, 1.37-3.54), $\operatorname{Der} f(\mathrm{aOR}, 2.23$; 95\% CI, 1.27-3.91), and Japanese Hop (aOR, 6.59; 95\% CI, 1.09-39.85). The independent risk factors for current AR on immunoCAP included more than 1 sensitization (aOR, 1.84; 95\% CI, 1.20-2.82) and sensitization to $\operatorname{Der} f(\mathrm{aOR}$, 1.96; 95\% CI, 1.27-3.03) or mugwort (aOR, 4.63; 95\% CI, 1.5114.23) (Table 4).

Table 2. Risk factors for current AR in preschool children

\begin{tabular}{|c|c|c|c|c|}
\hline Risk factors & $\mathrm{OR}(95 \% \mathrm{Cl})$ & $P$ value & $\mathrm{aOR} \mathrm{R}^{*}(95 \% \mathrm{Cl})$ & $P$ value \\
\hline \multicolumn{5}{|l|}{ Genetic factors } \\
\hline Parental asthma & $3.22(1.71-6.07)$ & $<0.001$ & $2.10(1.08-4.08)$ & 0.028 \\
\hline Parental AR & $2.88(2.01-4.14)$ & $<0.001$ & $2.25(0.92-5.52)$ & 0.077 \\
\hline Parental AD & $1.49(0.91-2.43)$ & 0.115 & $0.93(0.54-1.61)$ & 0.800 \\
\hline \multicolumn{5}{|l|}{ Past history } \\
\hline Past history of asthma diagnosis & $3.44(2.10-5.63)$ & $<0.001$ & $2.96(1.73-5.08)$ & $<0.001$ \\
\hline Wheezing, last 12 months & $1.89(1.13-3.16)$ & 0.015 & $2.09(1.21-3.59)$ & 0.008 \\
\hline Past history of $\mathrm{AD}$ diagnosis & $1.74(1.23-2.47)$ & 0.002 & $1.61(1.11-2.34)$ & 0.012 \\
\hline History of bronchiolitis before 2 years of age & $1.39(0.91-2.12)$ & 0.132 & $1.18(0.74-1.89)$ & 0.479 \\
\hline Breast milk feeding $>6$ months & $1.27(0.84-1.95)$ & 0.262 & $1.24(0.80-1.94)$ & 0.338 \\
\hline Use of antibiotics in infancy (>3 days) & $1.95(1.36-2.80)$ & $<0.001$ & $1.97(1.33-2.90)$ & $<0.001$ \\
\hline Cesarean-section delivery & $1.35(0.95-1.92)$ & 0.100 & $1.37(0.94-2.00)$ & 0.099 \\
\hline \multicolumn{5}{|l|}{ Environmental factors } \\
\hline Environmental tobacco smoking & $1.09(0.77-1.53)$ & 0.127 & $1.15(0.79-1.67)$ & 0.460 \\
\hline Current pet ownership & $0.63(0.19-2.12)$ & 0.451 & $0.66(0.19-2.29)$ & 0.508 \\
\hline Pet ownership in infancy & $0.85(0.37-1.94)$ & 0.323 & $0.80(0.34-1.86)$ & 0.600 \\
\hline Dog ownership in infancy & $0.96(0.42-2.21)$ & 0.928 & $0.90(0.38-2.12)$ & 0.810 \\
\hline Cat ownership in infancy & $1.17(0.13-10.51)$ & 0.891 & $1.00(0.11-9.30)$ & 0.996 \\
\hline Day care attendance before 1-year-old & $0.84(0.48-1.48)$ & 0.551 & $0.97(0.53-1.76)$ & 0.908 \\
\hline Having an older sibling & $0.56(0.39-0.79)$ & $<0.001$ & $0.52(0.35-0.75)$ & 0.001 \\
\hline Having a younger sibling & $1.09(0.76-1.57)$ & 0.633 & $1.11(0.77-1.64)$ & 0.561 \\
\hline History of remodeling, currently & $0.92(0.57-1.48)$ & 0.736 & $0.82(0.48-1.39)$ & 0.452 \\
\hline Indoor mold exposure during infancy & $1.69(1.18-2.40)$ & 0.004 & $1.67(1.15-2.43)$ & 0.007 \\
\hline Indoor mold exposure during previous last 12 months & $1.18(0.84-1.67)$ & 0.347 & $1.09(0.75-1.58)$ & 0.646 \\
\hline
\end{tabular}

$\mathrm{AR}$, allergic rhinitis; $\mathrm{OR}$, odds ratio; $\mathrm{OOR}$, adjusted odds ratio; $\mathrm{Cl}$, confidence interval; $\mathrm{AD}$, atopic dermatitis; $\mathrm{BMI}$, body mass index.

${ }^{*}$ Adjusted by age, sex, BMI, parental history of allergic diseases, and maternal education levels. 
Table 3. Associated positive sensitization on SPTs for the development of current $A R$

\begin{tabular}{lcccc}
\hline Risk factors & $\mathrm{OR}(95 \% \mathrm{Cl})$ & $P$ value & $\mathrm{aOR}^{*}(95 \% \mathrm{Cl})$ & $P$ value \\
\hline SPT any positive & $2.34(1.52-3.61)$ & $<0.001$ & $1.99(1.26-3.14)$ & 0.003 \\
Der $p$ & $2.56(1.63-4.02)$ & $<0.001$ & $2.23(1.37-3.54)$ & 0.001 \\
Derf & $2.62(1.54-4.46)$ & $<0.001$ & $2.23(1.27-3.91)$ & 0.005 \\
Alder & $0.86(0.10-7.42)$ & 0.890 & $0.77(0.09-6.84)$ & 0.815 \\
Birch & $1.44(0.15-13.93)$ & 0.755 & $1.14(0.11-11.4)$ & 0.913 \\
Mugwort & $2.90(0.48-17.53)$ & 0.247 & $0.81(0.08-7.97)$ & 0.856 \\
Japanese Hop & $6.59(1.09-39.85)$ & 0.040 & $3.57(0.47-27.37)$ & 0.221 \\
Dog & $2.19(0.65-7.39)$ & 0.207 & $2.14(0.53-8.60)$ & 0.285 \\
Cat & $4.35(0.61-31.22)$ & 0.143 & $4.56(0.61-34.30)$ & 0.140 \\
Egg white & $8.72(0.78-96.99)$ & 0.078 & $10.31(0.86-123.00)$ & 0.065 \\
Alternaria & $4.35(0.61-31.22)$ & 0.143 & $4.49(0.58-34.77)$ & 0.151 \\
Aspergillus & $4.32(0.27-69.64)$ & 0.302 & $2.73(0.16-45.29)$ & 0.485 \\
Ragweed & $4.33(0.27-69.64)$ & 0.302 & $5.83(0.35-96.80)$ & 0.219 \\
Oak & $1.08(0.12-9.71)$ & 0.865 & $0.81(0.09-7.58)$ & 0.852 \\
\hline
\end{tabular}

SPT, skin prick test; $\mathrm{AR}$, allergic rhinitis; $\mathrm{OR}$, odds ratio; aOR, adjusted odds ratio; Cl, confidence interval; Der p, Dermatophagoides pteronyssinus; Der f, Dermatophagoides farina; $\mathrm{BMI}$, body mass index.

${ }^{*}$ Adjusted by age, sex, BMl, parental history of allergic diseases, and maternal education levels.

Table 4. Associated positive serum-specific lgE test results for the development of current $A R$

\begin{tabular}{lllll}
\hline Risk factors & OR $(95 \% \mathrm{CI})$ & $P$ value & $\mathrm{aOR}^{*}(95 \% \mathrm{Cl})$ & $P$ value \\
\hline $\begin{array}{l}\text { Sensitization } \\
\quad 1 \text { allergens }\end{array}$ & $2.01(1.39-3.13)$ & $<0.001$ & $1.84(1.20-2.82)$ & 0.006 \\
Derf & $2.25(1.49-3.38)$ & $<0.001$ & $1.96(1.27-3.03)$ & 0.002 \\
Cat & $3.15(0.83-11.92)$ & 0.091 & $3.47(0.89-13.50)$ & 0.073 \\
Dog & $2.36(0.78-7.19)$ & 0.130 & $2.97(0.90-9.80)$ & 0.074 \\
Cockroach & $1.03(0.29-3.72)$ & 0.961 & $1.03(0.28-3.82)$ & 0.965 \\
Alternaria & $2.56(0.91-7.18)$ & 0.075 & $2.72(0.92-8.06)$ & 0.071 \\
Mugwort & $5.47(1.86-16.10)$ & 0.002 & $4.63(1.51-14.23)$ & 0.007 \\
Alder & $2.33(0.84-6.43)$ & 0.103 & $1.97(0.69-5.64)$ & 0.206
\end{tabular}

Positive specific lgE levels are defined as $\geq 0.35 \mathrm{kU} / \mathrm{L}$ for each allergen. IgE, immunoglobulin $\mathrm{E} ; \mathrm{AR}$, allergic rhinitis; OR, odds ratio; aOR, adjusted odds ratio; $\mathrm{Cl}$, confidence interval; Derf, Dermatophagoides farinae; $\mathrm{BMI}$, body mass index.

*Adjusted by age, sex, BMI, parental history of allergic diseases, and maternal education levels.

\section{Levels of FeNO and total serum IgE, and blood eosinophil} counts in current atopic AR patients without asthma or atopy

The geometric mean levels of FeNO were significantly higher in children with current atopic AR without asthma than in either control individuals or those with non-atopic rhinitis without asthma groups (12.43; 95\% CI, 10.07-15.49 vs 8.25; 95\% CI, 7.39-9.39, $P=0.001$ ). Furthermore, children with current atopic AR without asthma had significantly higher geometric mean

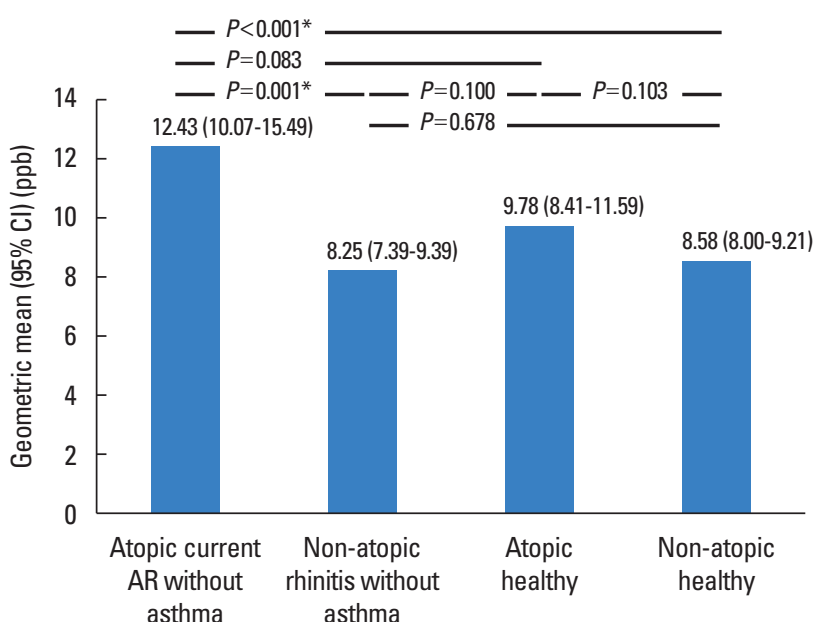

Fig. 2. FeNO levels among children having current atopic AR without asthma along with those in atopic and non-atopic healthy children. FeNO, fractional exhaled nitric oxide; AR, allergic rhinitis; $\mathrm{Cl}$, confidence interval. ${ }^{*} P<0.01$.

levels of FeNO compared with non-atopic healthy children (8.58; 95\% CI, 8.00-9.21, $P<0.001$ ). The levels of FeNO were higher in children with current atopic AR without asthma compared with those in atopic healthy children (12.43; 95\% CI, $10.07-15.49$ vs 9.78 ; $95 \%$ CI, 8.41-11.59, $P=0.083$ ) (Fig. 2).

Levels of total serum IgE (210.61; 95\% CI, 134.29-333.62 vs $55.70 ; 95 \% \mathrm{CI}, 44.70-70.10, P<0.001)$ and the percentages of blood eosinophils (5.58; 95\% CI, 4.39-6.82 vs 3.16; 95\% CI, 2.723.63, $P<0.001$ ) from children having current atopic AR without asthma were higher than those with non-atopic rhiinitis without asthma (Supplementary Figs. 1 and 2). The total serum IgE levels tended to be higher in children with current atopic AR without asthma compared with those in the atopic healthy children (210.61; 95\% CI, 134.29-333.62 vs 149.90; 95\% CI, 114.43200.34, $P=0.193)$. Children with current atopic AR without asthma had a significantly higher geometric mean blood eosinophil percentage compared with those from the atopic healthy children (3.93; 95\% CI, 3.35-4.62, $P=0.015)$.

\section{Cutoff values for FeNO, total serum IgE, and blood eosinophil percentages to define atopic current $A R$}

To define cutoff values for FeNO to differentiate children atopic current AR from atopic healthy children, we constructed a receiver operation characteristic (ROC) curve for the diagnosis of current atopic AR. A FeNO $>7.5$ ppb yielded a sensitivity of $86.4 \%$, a specificity of $47.7 \%$, and the area under the ROC curve (AUC) of 0.714 (95\% CI, 0.600-0.829). Additionally, we calculated optimal cutoff values for serum IgE of $>86.1 \mathrm{kU} / \mathrm{L}$, with a sensitivity of $83.2 \%$, a specificity of $71.5 \%$, and an AUC of 0.835 (95\% CI, 0.811-0.858), as well as a blood eosinophil frequency of $>2.5 \%$, sensitivity of $66.1 \%$, specificity of $68.1 \%$, and an AUC of 0.706 (95\% CI, 0.674-0.738; Table 5). A significant linear correlation between FeNO, total serum IgE levels, and blood eo- 
Table 5. Cutoff values for FeNO, serum IgE, and blood eosinophil levels in children with current atopic AR without asthma

\begin{tabular}{lccc}
\hline & Cut-off value & Sensitivity (\%) & Specificity (\%) \\
\hline FeNO (ppb) & $>7.5$ & 86.4 & 47.7 \\
Serum lgE (kU/L) & $>86.1$ & 83.2 & 71.5 \\
Blood eosinophil (\%) & $>2.5$ & 66.1 & 68.1 \\
\hline
\end{tabular}

FeNO, fractional exhaled nitric oxide; lgE, immunoglobulin $\mathrm{E}$; $A R$, allergic rhinitis.

sinophil percentage was observed $(\mathrm{r}=0.244, P<0.001$ for serum IgE and $\mathrm{r}=0.296, P<0.001$ for blood eosinophilia; Supplementary Figs. 3A and B).

\section{DISCUSSION}

Our current general population-based study demonstrated the prevalence of AR and the discriminative value of FeNO for the diagnosis of AR in preschool-aged children in Seoul, Korea. In the present study, the prevalence of current AR, atopic current AR, AR symptoms ever in lifetime, and atopic AR symptoms ever in lifetime in preschool-aged children were $17.0 \%$, $4.7 \%, 43.8 \%$, and $8.4 \%$, respectively, which indicates that AR is not uncommon in preschool-aged children. A parental history of asthma, a past history of asthma, $\mathrm{AD}$, indoor mold exposure, antibiotic use during infancy, and atopy increased the risk of current AR, whereas the presence of an older sibling reduced the risk of current AR. Sensitization to Der $p$, Der $f$, and Japanese hop was associated with an increased risk of current AR in preschool-aged children. In this study, we excluded children with asthma and divided the current AR group into 2 subgroups based on the presence of atopy. The levels of FeNO were significantly higher in children with current atopic AR without asthma (12.43 ppb; 95\% CI, 10.07-15.49) compared those with nonatopic rhinitis without asthma (8.25 ppb; 95\% CI, 7.39-9.39; $P=0.001)$ or atopic healthy children (9.78 ppb; $95 \% \mathrm{CI}, 8.41-$ 11.59), although the latter difference was not statistically significant $(P=0.083)$. Therefore, we should suspect $A R$ when atopic preschool children complain of AR symptoms and present with high levels of FeNO.

Some children may only exhibit significant symptoms with a combination of both allergic inflammation and coexisting viral infection. Additionally, it can be difficult to differentiate AR from other common diseases, such as non-allergic rhinitis (NAR) and chronic rhinosinusitis, as these diseases share similar symptoms with AR in young children. These similarities are particularly common in preschool-aged children. ${ }^{18}$ We diagnosed current $\mathrm{AR}$ as nasal symptoms and also used physician diagnoses to reduce potential errors in the misdiagnosis of AR. Furthermore, the findings of our study are important, as they combine a questionnaire survey and clinical diagnoses with the results of SPT in a large randomly selected preschool children with AR.
The reported prevalence of AR in preschool-aged children is diverse and varies by region. ${ }^{19}$ This finding might be partially attributed to misdiagnoses and be related to the physician's experience in the diagnosis of AR. However, the prevalence of AR has been progressively increasing worldwide, while the age of onset is decreasing. ${ }^{19}$ In a birth cohort study carried out in Sweden in 2003, the frequency of 4.5-year-old children who reported symptoms that were compatible with AR during the previous year was $5.5 \%(246 / 4,465)$, and the frequency of doctor-diagnosed AR based on symptoms during the last year was $1.7 \%$ $(75 / 4,465) .{ }^{20}$ In a study conducted in China using an ISAAC questionnaire, the prevalence of $\mathrm{AR}$, which was defined as nasal symptoms and atopy on SPTs, was $10.8 \%$ in 3- to 6-year-old children, ${ }^{9}$ a rate that was similar to the prevalence of atopic AR symptoms in an individual's lifetime (8.4\%) observed in our study. In a study carried out in Seoul, Korea using an ISAAC questionnaire, the prevalences of $\mathrm{AR}$ ever, $\mathrm{AR}$ in the last 12 months, AR diagnosis ever, and treatment in the last 12 months were $31.8 \%, 26.2 \%, 16.2 \%$, and $12.9 \%$, respectively, in children who were 2-7 years old in 2009. ${ }^{21}$ Furthermore, in a study of 3to 6-year-old preschool children in the Gyeonggi Province of Korea in 2009, the prevalence of AR ever was $44.2 \%$, while that of AR symptoms in the last 12 months was $40.7 \%$, that of AR diagnosis ever was $34.5 \%$, and that of treatment in the last 12 months was $28.0 \%{ }^{22}$

The findings of the aforementioned studies were similar to those of our study, which used the ISAAC questionnaire, as the prevalences of AR symptoms ever in life, AR symptoms in the 12 months, AR diagnosis in the life time and AR treatment in the life time were $43.8 \%, 38.5 \%, 23.8 \%$, and $18.6 \%$ respectively. Differences in the prevalence of AR between our report and earlier studies from China might be in part attributed to differences in the definition of AR combined with atopy in SPTs. Additionally, differences in environmental factors according to regions may affect the prevalence of AR. However, the use of a questionnaire method may result in a the high probability of overestimation. The prevalence of atopy for SPTs is low, especially in preschool children; therefore, application results based on SPTs could result in an underestimated diagnosis of AR. Thus, further studies will be required to accurately determine the prevalence of AR in a large study using a unified definition of AR. Moreover, considering that conducting SPTs on preschool children was particularly difficult in this study, a non-invasive FeNO test may aid in diagnoses of AR in preschool children. However, the prevalence of AR is not uncommon in preschool children. Hence, we must further characterize when AR develops, determine how to diagnose $\mathrm{AR}$, and assess whether cases of AR in preschool children are increasing, so that followup studies about the pathogenesis of AR are needed.

In our study, environmental factors that we identified as independent risk factors for AR included the use of antibiotics in infancy and exposure to household mold during infancy. A pro- 
tective effect of having an older sibling on AR in preschool-aged children was observed, which was consistent with an inverse association between the number of siblings and hay fever.. ${ }^{23,24}$ The use of antibiotics in early life is known to lead to alterations in the intestinal flora and increase the risk of childhood atopy and asthma. ${ }^{23-25}$ Therefore, our data support the hygiene hypothesis that microbiome naturally colonizing in our body may affect the development of human immune system and the development of allergic disease. Especially during pregnancy and within 3 years after birth, the composition of the microorganisms in the intestinal microbiome is affected by the delivery method of the infant as well as differences in diet composition. ${ }^{26}$ Antibiotic use during infancy induces changes in the composition of the intestinal microbial strain, and this is associated with suppressed T helper 1 (Th1)-type immunity after normal birth as well as progressively increased serum IgE levels and regulatory $\mathrm{T}$ cells, which are known to suppress $\mathrm{T}$ helper 2 (Th2)-type immune responses. ${ }^{27,28}$

In our study, Der $f$ and $\operatorname{Der} p$ were found to be independent risk factors for allergic sensitization on SPT and CAP in preschool-aged children with current AR. Exposure to indoor allergens at home is a common cause of perennial AR, especially in young children who spend most of their time indoors. Therefore, in children with allergic sensitization to house dust mite allergens, avoidance of house dust mites is always a critical component of a management strategy for AR in preschool-aged children. ${ }^{10}$ Additionally, mugwort was identified as a major independent risk factor for AR in preschool-aged children.

A key issue is the relationship between AR and FeNO levels. FeNO analysis is often used to assess eosinophilic airway inflammation in bronchial asthmatic patients. Many studies have shown increased levels of FeNO in adults with $\mathrm{AR} .{ }^{19}$ Although the literature on FeNO measurements in children with AR is less extensive, FeNO has been reported to be increased in children with $A R .{ }^{29}$ In China, in a case-control study with age- and gender-matched children based on a lager cross-sectional general population survey on asthma, AR and AD among 9- to 11-year-old school-aged children with physician-diagnosed AR without asthma had significantly elevated levels of FeNO $(25.8 \pm 2.1 \mathrm{ppb})$ compared with the control group $(13.3 \pm 1.6$ $\mathrm{ppb})$, which included children without physician-diagnosed asthma, $\mathrm{AR}$, or $\mathrm{AD}(P=0.008)$. Although they did not control whether the patients had atopy or not, they showed that AR could independently cause an increase in FeNO levels in Chinese school- aged children. ${ }^{30}$ Although several studies have assessed FeNO levels in school-children with asthma or $\mathrm{AR}^{30,31}$ limited data are available about FeNO levels in preschool-aged children. The FeNO level is low at younger age $\mathrm{e}^{32}$ and a study that suggested FeNO level of recurrent wheezers in preschool children was approximately 10-15 ppb, which is lower than school-aged children's levels. ${ }^{33}$ Our study focused on the presentation of FeNO levels in preschool children with AR who were 3-7 years old and controlled for the confounding factor of asthma, which is one of the most powerful determinants of increased FeNO levels. We believe that further studies on FeNO with preschool children will be required to compare the results with our study, rather than using the FeNO level of the older children's reference values.

Most previous reports of the prevalence of AR have been based on a questionnaire survey alone, which may have resulted in an overestimation of the prevalence (up to 50\%) of AR. ${ }^{34}$ Similar to other studies, our findings were based on a questionnaire survey. Therefore, as we diagnosed current AR based on a questionnaire, both recall and selection bias may exist. Additionally, reporting bias might have exist because clinicians did not directly examine and follow up the current AR patients. To evaluate the actual prevalence of AR, we classified current atopic $\mathrm{AR}$ and non-atopic rhinitis to differentiate between rhinitis with and without sensitization to allergens. Another limitation of our study was its cross-sectional design, which limited our ability to determine causation. However, our study was population-based, which is a major strength that allows for an estimation of the population attributable risk of multiple factors. Furthermore, it is difficult to compare the prevalence of current AR calculated herein with previous studies because of differences between the study population groups, which were from different areas. Despite these limitations, our current findings merit attention because limited data are available on the prevalence of AR in preschool-aged children. An additional strength of our study was that we performed the FeNO test among preschool children who were suspected AR cases using a non-invasive biomarker.

In conclusion, the prevalence of current $\mathrm{AR}$ in preschool-aged children was $17.0 \%$, indicating that the onset of AR can be significant at younger age. Furthermore, levels of FeNO were higher in children who had current atopic AR, which suggests that FeNO levels can be a useful diagnostic marker of current AR in preschool-aged children. Future surveillance efforts that follow a well-designed large patient cohort will be required to quantitatively assess the prevalence of AR and its associated risk factors in preschool-aged children. Furthermore, prospective, large-scale, randomized control trials will be needed to better characterize and understand the role of FeNO in the clinical care of young children with AR.

\section{ACKNOWLEDGMENTS}

This study was supported by grant of the Korean Health Technology R \& D Project, Ministry of Health and Welfare, Republic of Korea (A092076 and HI13C1674).

\section{REFERENCES}

1. Chen Y, Wong GW, Li J. Environmental exposure and genetic pre- 
disposition as risk factors for asthma in China. Allergy Asthma Immunol Res 2016;8:92-100.

2. Yoon HI. Respiratory review of 2014: asthma. Tuberc Respir Dis (Seoul) 2014;77:237-42.

3. Meyers DA, Bleecker ER, Holloway JW, Holgate ST. Asthma genetics and personalised medicine. Lancet Respir Med 2014;2:405-15.

4. Vahedi G, Richard AC, O'Shea JJ. Enhancing the understanding of asthma. Nat Immunol 2014;15:701-3.

5. Tizaoui K, Kaabachi W, Hamzaoui K, Hamzaoui A. Association of single nucleotide polymorphisms in toll-like receptor genes with asthma risk: a systematic review and meta-analysis. Allergy Asthma Immunol Res 2015;7:130-40.

6. Zhao CN, Fan Y, Huang JJ, Zhang HX, Gao T, Wang C, et al. The association of GSDMB and ORMDL3 gene polymorphisms with asthma: a meta-analysis. Allergy Asthma Immunol Res 2015;7:17585.

7. Huang J, Wu S, Barrera J, Matthews K, Pan D. The hippo signaling pathway coordinately regulates cell proliferation and apoptosis by inactivating Yorkie, the Drosophila Homolog of YAP. Cell 2005;122: 421-34.

8. Angus L, Moleirinho S, Herron L, Sinha A, Zhang X, Niestrata M, et al. Willin/FRMD6 expression activates the hippo signaling pathway kinases in mammals and antagonizes oncogenic YAP. Oncogene 2012;31:238-50.

9. Zhao B, Wei X, Li W, Udan RS, Yang Q, Kim J, et al. Inactivation of YAP oncoprotein by the hippo pathway is involved in cell contact inhibition and tissue growth control. Genes Dev 2007;21:2747-61.

10. Alarcón C, Zaromytidou AI, Xi Q, Gao S, Yu J, Fujisawa S, et al. Nuclear CDKs drive Smad transcriptional activation and turnover in BMP and TGF $\beta$ pathways. Cell 2009;139:757-69.

11. Strano S, Munarriz E, Rossi M, Castagnoli L, Shaul Y, Sacchi A, et al. Physical interaction with Yes-associated protein enhances p73 transcriptional activity. J Biol Chem 2001;276:15164-73.

12. Vassilev A, Kaneko KJ, Shu H, Zhao Y, DePamphilis ML. TEAD/ TEF transcription factors utilize the activation domain of YAP65, a Src/Yes-associated protein localized in the cytoplasm. Genes Dev 2001;15:1229-41.

13. Mahoney JE, Mori M, Szymaniak AD, Varelas X, Cardoso WV. The hippo pathway effector Yap controls patterning and differentiation of airway epithelial progenitors. Dev Cell 2014;30:137-50.

14. Lange AW, Sridharan A, Xu Y, Stripp BR, Perl AK, Whitsett JA. Hippo/Yap signaling controls epithelial progenitor cell proliferation and differentiation in the embryonic and adult lung. J Mol Cell Biol 2015;7:35-47.

15. Ungvári I, Hullám G, Antal P, Kiszel PS, Gézsi A, Hadadi É, et al. Evaluation of a partial genome screening of two asthma susceptibility regions using bayesian network based bayesian multilevel analysis of relevance. PLoS One 2012; 7:e33573.

16. Ungvári I, Hadadi E, Virág V, Bikov A, Nagy A, Semsei ÁF, et al. Implication of BIRC5 in asthma pathogenesis. Int Immunol 2012;24: 293-301.

17. Wenzel SE. Asthma: defining of the persistent adult phenotypes. Lancet 2006;368:804-13.

18. Antal P, Millinghoffer A, Hullám G, Hajós G, Sárközy P, Gézsi A, et al. Bayesian, systems-based, multilevel analysis of associations for complex phenotypes: from interpretation to decisions. In: Sinoquet C, Mourad R, editors. Probabilistic graphical models for genetics, genomics, and postgenomics. Oxford: Oxford University Press; 2014.
318-62.

19. Antal P, Millinghoffer A, Hullám G, Hajós G, Szalai C, Falus A. A bioinformatic platform for a bayesian, multiphased, multilevel analysis in immunogenomics. In: Flower DR, Davies M, Ranganathan S, editors. Bioinformatics for immunomics. New York (NY): Springer; 2010. 157-85.

20. Hullám G, Antal P, Szalai C, Falus A. Evaluation of a Bayesian model-based approach in GA studies. JMLR Workshop Conf Proc 2010; 8:30-43.

21. Antal P, Millinghoffer A, Hullám G, Szalai C, Falus AA. Bayesian view of challenges in feature selection: multilevel analysis, feature aggregation, multiple targets, redundancy and interaction. JMLR Workshop Conf Proc 2008;4:74-89.

22. Gézsi A, Lautner-Csorba O, Erdélyi DJ, Hullám G, Antal P, Semsei ÁF, et al. In interaction with gender a common CYP3A4 polymorphism may influence the survival rate of chemotherapy for childhood acute lymphoblastic leukemia. Pharmacogenomics J 2015; 15:241-7.

23. Lautner-Csorba O, Gézsi A, Erdélyi DJ, Hullám G, Antal P, Semsei ÁF, et al. Roles of genetic polymorphisms in the folate pathway in childhood acute lymphoblastic leukemia evaluated by Bayesian relevance and effect size analysis. PLoS One 2013;8:e69843.

24. Lautner-Csorba O, Gézsi A, Semsei AF, Antal P, Erdélyi DJ, Schermann $\mathrm{G}$, et al. Candidate gene association study in pediatric acute lymphoblastic leukemia evaluated by Bayesian network based Bayesian multilevel analysis of relevance. BMC Med Genomics 2012;5:42.

25. Chan TK, Loh XY, Peh HY, Tan WN, Tan WS, Li N, et al. House dust mite-induced asthma causes oxidative damage and DNA doublestrand breaks in the lungs. J Allergy Clin Immunol 2016;138:84-96. el.

26. Mao B, Gao Y, Bai Y, Yuan Z. Hippo signaling in stress response and homeostasis maintenance. Acta Biochim Biophys Sin (Shanghai) 2015;47:2-9.

27. Piccolo S, Dupont S, Cordenonsi M. The biology of YAP/TAZ: hippo signaling and beyond. Physiol Rev 2014;94:1287-312.

28. Yin MX, Zhang L. Hippo signaling in epithelial stem cells. Acta Biochim Biophys Sin (Shanghai) 2015;47:39-45.

29. Moleirinho S, Patrick C, Tilston-Lünel AM, Higginson JR, Angus L, Antkowiak M, et al. Willin, an upstream component of the hippo signaling pathway, orchestrates mammalian peripheral nerve fibroblasts. PLoS One 2013;8:e60028.

30. Jeffery PK, Wardlaw AJ, Nelson FC, Collins JV, Kay AB. Bronchial biopsies in asthma. An ultrastructural, quantitative study and correlation with hyperreactivity. Am Rev Respir Dis 1989;140:1745-53.

31. Beasley R, Roche WR, Roberts JA, Holgate ST. Cellular events in the bronchi in mild asthma and after bronchial provocation. Am Rev Respir Dis 1989;139:806-17.

32. Laitinen LA, Heino M, Laitinen A, Kava T, Haahtela T. Damage of the airway epithelium and bronchial reactivity in patients with asthma. Am Rev Respir Dis 1985;131:599-606.

33. Boulet LP, O'Byrne PM. Asthma and exercise-induced bronchoconstriction in athletes. N Engl J Med 2015;372:641-8.

34. Weiler JM, Anderson SD, Randolph C, Bonini S, Craig TJ, Pearlman DS, et al. Pathogenesis, prevalence, diagnosis, and management of exercise-induced bronchoconstriction: a practice parameter. Ann Allergy Asthma Immunol 2010;105:S1-47. 